\title{
Índice de competitividade regional municipal: uma aplicação simplificada em municípios brasileiros
}

\author{
Leonardo de Oliveira Dresch ${ }^{1}$ \\ Adriano Marcos Rodrigues Figueiredo ${ }^{2}$ \\ Mayra Batista Bitencourt Fagundes ${ }^{3}$
}

Submissão: 30/09/2021

Aceite: $18 / 10 / 2021$

\begin{abstract}
Resumo
A competitividade regional, dada pela capacidade das regiões em gerar riquezas e revertê-la como melhoria da qualidade de vida para seus habitantes, é amplamente discutida por acadêmicos e decisores políticos. $O$ artigo buscou aprofundar-se em como mensurar e classificar a competitividade regional em municípios brasileiros? Foi utilizada uma adaptação do Índice de Competitividade Regional Municipal (ICRM) proposto por Dresch (2021), denominada como ICRM-A, restringindo-se a 8 indicadores do ano de 2010, que foi aplicado em 5.565 municípios brasileiros. Os resultados apontam para uma heterogeneidade inter-regional significativa em relação a competitividade, com a região sul e sudeste possuindo a maior parte dos municípios classificados com ICRM Alto e Muito Alto, $76 \%$ e $91 \%$ respectivamente, enquanto nas regiões norte e nordeste estão concentrados $92,99 \%$ do total de municípios com Baixo ICRM-A.
\end{abstract}

Palavras-chave: Competitividade regional; Municípios brasileiros; índice de competitividade.

\section{Municipal regional competitiveness index: a simplified application for Brazilian municipalities}

\begin{abstract}
Regional competitiveness, given by the regions' ability to generate wealth and revert it to improve the quality of life for its inhabitants, is widely discussed by academics and policy makers. Did the article seek to deepen how to measure and classify regional competitiveness in Brazilian municipalities? An adaptation of the Municipal Regional Competitiveness Index (ICRM) proposed by Dresch (2021), called ICRM-A, was used, restricting itself to 8 indicators for the year 2010, which was applied in 5,565 Brazilian municipalities. The results point to a significant interregional heterogeneity in relation to competitiveness, with the south and southeast regions having most of the municipalities classified with ICRM Alto and Very High, 76\% and 91\% respectively, while in the north and northeast regions are concentrated $92.99 \%$ of the total municipalities with Low ICRM-A.
\end{abstract}

Key words: Regional competitiveness; Brazilian municipalities; competitiveness index.

\section{Introdução}

O Brasil ocupa um território superior a 8,5 milhões de quilômetros quadrados de extensão. Está localizado ao sul do continente americano fazendo fronteira com o Uruguai ao sul; Argentina e Paraguai a sudoeste; Bolívia e Peru a oeste; Colômbia a noroeste e Venezuela,

\footnotetext{
${ }^{1}$ Doutorado em Administração (UFMS). https://orcid.org/0000-0001-7161-9693. Email:adm.leonardo.dresch@gmail.com

2 Doutorado e Pós-Doutorado em Economia Aplicada (UFV). Professor do Programa de Pós-Graduação de Administração e Contabilidade da UFMS. https://orcid.org/0000-0002-3677-1291. Email:adriano.figueiredo@gmail.com

${ }^{3}$ Doutorado em Economia Aplicada (UFV). Professora do Programa de Pós-Graduação de Administração e Contabilidade da UFMS. https://orcid.org/0000-0003-3961-2330. E-mail:bitencourtmayra@gmail.com
} 
Suriname, Guyana e Guyana Francesa ao norte. São mais de 23 mil quilômetros de fronteiras. Esse país, de dimensões continentais, guarda em seu território uma ampla diversidade natural e cultural.

A desigualdade entre as regiões, especialmente a pobreza, é alvo de atenção do Estado, que determina na Constituição Federal em seu artigo terceiro (art. 3.ํ), do parágrafo terceiro (§ 3.), que é objetivo fundamental da República Federativa do Brasil "erradicar a pobreza e a marginalização e reduzir as desigualdades sociais e regionais". 0 artigo 170 , destaca ainda nos princípios gerais da atividade econômica, em seu parágrafo sétimo, a busca pela "redução das desigualdades regionais e sociais" (BRASIL, 2021, on-line).

A competitividade é uma das mais poderosas forças de uma sociedade para tornar as coisas melhores em vários campos do empenho humano (PORTER, 2008). Entretanto, em se falando de competitividade regional, deve-se abandonar o conceito de soma zero, em que existem sempre perdedores e vencedores, mas ver a competitividade como a busca das regiões por ofertar uma plataforma adequada para se operar em altos níveis de produtividade (muito diferente do tipo de concorrência direta realizada pelas empresas) (HUGGINS; THOMPSON, 2017)

A competitividade regional é considerada como a "capacidade de uma região particular em atrair e manter as empresas com participação de mercado estáveis ou crescentes, enquanto mantém estáveis ou crescentes padrões de vida para aqueles que participam disso" (STORPER, 1997, p. 264, tradução nossa). O aparato teórico desenvolvido especialmente por geógrafos econômicos e economistas espaciais, sob a égide da competitividade regional, busca em seu cerne explicar o desenvolvimento desigual em contextos regionais (HUGGINS; THOMPSON, 2017).

Existem muitos rankings desenvolvidos atualmente para comparar regiões, via benchmarking, quanto aos seus níveis de competitividade. Alguns dos exemplos mais notáveis são o Global Competitiveness Index (CGI) desenvolvido e mantido pelo Fórum Econômico Mundial, o European Regional Competitiveness Index (EU RCI) da Comissão Européia, e, o Word Competitiveness Ranking (WCR) do Institute for Management Development (IMD). Em comum todos eles congregam, em sua estrutura, uma diversidade de variáveis e indicadores que possuem potencial para explicar a competitividade das regiões.

O problema ao qual esse artigo buscou abordar foi como mensurar e classificar a competitividade regional em municípios brasileiros? Para tanto foi utilizado o Índice de Competitividade Regional Municipal (ICRM) de Dresch (2021) adaptado para lidar com a 
complexidade e abrangência de se trabalhar com todos os municípios do Brasil. A proposição do ICRM adaptado, denominado ICRM-A, possibilitou uma análise exploratória da distribuição dos municípios conforme seus respectivos potenciais competitivos entre as diversas regiões brasileiras.

O artigo está dividido em: i) introdução; ii) competitividade regional; iii) metodologia; iv) resultados e discussões; e, v) considerações finais. O trabalho poderá ser precursor de mais estudos e análises sobre a competitividade regional no Brasil e propostas de políticas públicas voltadas a redução da desigualdade entre as regiões.

\section{Competitividade regional}

As duas palavras do próprio conceito de competitividade regional são fontes de dúvidas e requerem explicações ampliadas. A noção de região, por exemplo, pode significar unidades supra-nacionais e/ou unidades subnacionais. Um grupo de países ligados por uma relação geográfica que compartilha características comuns, objetivos econômicos, instituições e regras de comportamento, ou a noção de região pode se referir a uma unidade estatística, ou ainda a uma unidade administrativa dentro de um país ou a unidades dentro de um país que correspondam à sua estrutura regional geográfica, histórica e cultural (BOROZAN,2008).

A competitividade é também um termo difícil, confuso e muitas vezes ambíguo, que levanta mais dúvidas que respostas. A compreensão do seu significado deve passar pela diferenciação entre três níveis de competitividade: micro, meso e macro. A competitividade microeconômica é definida como a capacidade de uma empresa em competir com sucesso em um mercado (sendo eficiente e eficaz), crescendo e obtendo lucro no longo prazo de forma responsável junto à comunidade local. A competitividade macroeconômica, ou nacional, parte da premissa de que os governos podem criar ambientes favoráveis para operação das empresas. Entre os níveis micro e macro da competitividade, existe a competitividade regional, que é vista como: i) em nível microeconômico, a que atribui aos decisores políticos o papel de criar condições de mercado sólidas, focando na produtividade de uma região como determinada pela produtividade de suas firmas (PORTER, 2003); ii) em nível macroeconômico, em que os locais devem manter condições que permitam a criação de valor agregado sustentável, melhoria do padrão de vida e o bem-estar de seus cidadãos (BOROZAN, 2008).

A mensuração da competitividade é frequentemente confundida com a questão do desempenho econômico, apesar de inegável a relação destes, não deve ser limitada. Aiginger 
(2006) aponta duas perspectivas que podem tornar a questão da mensuração da competitividade regional mais clara. Trata-se de uma métrica através dos resultados da competitividade (crescimento e desenvolvimento econômico) e outra através da produção e elementos associados. A primeira, bem-estar ou resultado da competitividade (output), é função de: Y é renda per capita, S que são indicadores sociais e distributivos, e, E que são os indicadores ecológicos. A métrica referente a produção ou entrada da competitividade (input), é função de: K é capital físico, Lé trabalho, TFP é a produtividade total dos fatores que representa o progresso técnico, C são as capacidades (competências), I são os efeitos institucionais, e, T é a confiança.

Segundo Annoni e Dijkstra (2017), medir a competitividade regional é um passo importante para informar aos decisores políticos sobre as estratégias regionais de desenvolvimento. A dimensão regional seria importante pois muitos fatores de competitividade são influenciados, ou mesmo determinados, pelas autoridades regionais e municipais. Para Aiginger (2006), a vantagens dos rankings de competitividade baseados em uma multiplicidade de indicadores, é que eles medem um amplo espectro de aspectos econômicos, o que reduz erros e distorções nos resultados que acontecem quando somente alguns poucos aspectos são considerados. Por outro lado, indicadores grandes frequentemente não possuem um conceito claro e por vezes combinam e misturam coisas que deveriam ser avaliadas separadamente (e.g. indicadores de resultado com os de produção).

\section{Metodologia}

Dresch (2021), em sua tese de doutorado, propôs um método para mensurar, classificar e analisar a competitividade regional municipal desenvolvendo dois instrumentos. Um para prospectar, elencar, organizar e avaliar as variáveis descritoras da competitividade regional municipal e o outro para analisar esse conjunto de características em relação ao recorte regional predeterminado. O teste empírico aconteceu nos 79 municípios de Mato Grosso do Sul, e durante o emprego deste, foi desenvolvido e aplicado o Índice de Competitividade Regional Municipal (ICRM) originalmente contemplando 52 variáveis explicativas.

O ICRM é baseado em medidas separatrizes. Segundo Spiegel (1993), o conjunto de dados organizado por ordem de grandeza, tem em seu valor central (ou na média aritmética dos dois valores centrais), a mediana, que divide todo o conjunto em duas partes iguais. Por extensão ao conceito de mediana, podemos pensar em valores que dividiriam o conjunto de dados em quatro partes iguais (quartis), ou dez partes iguais (decis) ou mesmo em cem partes iguais (percentis). 
O primeiro quartil (Q1) separa os $25 \%$ inferiores, o segundo quartil (Q2) é a mediana e o terceiro quartil (Q3) separa os $75 \%$ inferiores dos $25 \%$ superiores.

Conforme a posição do município referente a variável explicativa observada quanto ao conjunto de dados organizado em quartis, é atribuída pontuação conforme o seguinte critério: mínimo - 0 pontos; (Q1) primeiro - 1 ponto, (Q2) segundo - 2 pontos; (Q3) terceiro - 3 pontos; e, máximo - 4 pontos. Caso a relação da variável com a competitividade regional seja classificada como inversa, como é o caso da proporção de pobres (PMPOB), as pontuações são invertidas.

O ICRM original contemplou mais de 4.000 dados (52 variáveis explicativas de 79 municípios). A diversidade de fontes consultadas originalmente, 14, algumas com abrangência estadual, exigiu uma simplificação para aplicação nos 5.650 municípios brasileiros, do contrário seriam mais de 293 mil dados. Uma opção coerente e viável foi restringir o ICRM aos dois primeiros grupos do método proposto por Dresch (2021), respectivamente básico e eficiência, incluindo os oito subgrupos que os compõem: i) instituições; ii) estabilidade macroeconômica; iii) infraestrutura; iv) saúde; v) educação básica; vi) educação superior; vii) eficiência do mercado de trabalho; e, viii) tamanho do mercado. O terceiro grupo, inovação, e seus subgrupos prontidão tecnológica, sofisticação de negócios e inovação, não foram considerados em virtude da heterogeneidade elevada entre os municípios que acarretaria a ausência de dados para a maioria, além de exigir busca em diversas fontes diferentes. O quadro 1 consolida as variáveis explicativas selecionadas e as suas fontes.

Quadro 1 - Variáveis explicativas selecionadas para o ICRM simplificado.

\begin{tabular}{|c|c|c|c|}
\hline Subgrupo ou pilar & Variável & Sigla & Fonte \\
\hline 1. Instituições & Produto Interno Bruto & PIB & IBGE - Sidra \\
\hline $\begin{array}{l}\text { 2. Estabilidade } \\
\text { macroeconômica }\end{array}$ & Renda per capita média & RDPC & $\begin{array}{l}\text { PNUD - Atlas do } \\
\text { Desenvolvimento } \\
\text { Humano no Brasil }\end{array}$ \\
\hline 3. Infraestrutura & $\begin{array}{l}\text { Percentual da população que vive } \\
\text { em domicílios com energia elétrica }\end{array}$ & T_LUZ & PNUD \\
\hline 4. Saúde & Esperança de vida ao nascer & ESPVIDA & PNUD \\
\hline 5. Educação básica & $\begin{array}{l}\text { Expectativa de anos de estudo aos } \\
18 \text { anos de idade }\end{array}$ & E_ANOSESTUDO & PNUD \\
\hline 6. Educação superior & $\begin{array}{l}\text { Percentual da população de } 25 \\
\text { anos ou mais com superior } \\
\text { completo }\end{array}$ & T_SUPER25M & PNUD \\
\hline $\begin{array}{l}\text { 7. Eficiência do } \\
\text { mercado de trabalho }\end{array}$ & $\begin{array}{l}\text { Percentual de ocupados de } 18 \text { anos } \\
\text { ou mais que são empregadores }\end{array}$ & EMP & PNUD \\
\hline $\begin{array}{l}\text { 8. Tamanho do } \\
\text { mercado }\end{array}$ & Proporção de pobres & PMPOB & PNUD \\
\hline
\end{tabular}

Fonte: Elaborado pelos autores. 
1. Instituições - Optou-se pelo Produto Interno Bruto de 2010, disponível na tabela 5938 do IBGE Sidra, por compreender que a riqueza gerada em um município é uma proxy das suas receitas públicas e do tamanho e estrutura das suas instituições. É uma dimensão complicada de análise objetiva e diversas métricas de competitividade regional buscam painéis com especialistas para desenvolver uma avaliação subjetiva (baseada em percepção) da eficiência das instituições do local avaliado.

2. Estabilidade macroeconômica - Renda per capita média 2010, calculada pelo Atlas do Desenvolvimento Humano do PNUD, é dada pela "razão entre o somatório da renda de todos os indivíduos residentes em domicílios particulares permanentes e o número total desses indivíduos" tendo por referência valores em reais para agosto de 2010 (PNUD, 2010, n.p). A renda relativizada ajuda a impedir que valores absolutos obscureçam municípios menores, além de relacionar-se, de uma forma geral, a uma considerável parte do dinamismo econômico das regiões.

3. Infraestrutura - Percentual da população que vive em domicílios com energia elétrica do ano de 2010, também calculado pelo Atlas do Desenvolvimento Humano do PNUD, é dado pela "razão entre a população que vive em domicílios particulares permanentes com iluminação elétrica e a população total residente em domicílios particulares permanentes multiplicado por 100" considerando a energia proveniente ou não em rede geral, com ou sem medidor (PNUD, 2010, n.p). A iluminação elétrica é tida como uma proxy da infraestrutura disponível no município.

4. Saúde - Esperança de vida ao nascer em 2010, é igual ao "número médio de anos que as pessoas deverão viver a partir do nascimento, se permanecerem constantes ao longo da vida o nível e o padrão de mortalidade por idade prevalecentes no ano do Censo" (PNUD, 2010, n.p).

5. Educação básica - Expectativa de anos de estudo aos 18 anos de idade em 2010, é calculada a partir do "número médio de anos de estudo que uma geração de crianças que ingressa na escola deverá completar ao atingir 18 anos de idade, se os padrões atuais se mantiverem ao longo de sua vida escolar" (PNUD, 2010, n.p).

6. Educação superior - Percentual da população de 25 anos ou mais com ensino superior completo 2010, calculado através da "razão entre a população de 25 anos ou mais de idade que concluiu pelo menos a graduação do ensino superior e o total de pessoas nesta faixa etária multiplicado por 100" (PNUD, 2010, n.p).

7. Eficiência do mercado de trabalho - Percentual de ocupados de 18 anos ou mais que são empregadores 2010, dado pela "razão entre o número de empregadores de 18 anos ou mais 
de idade e o número total de pessoas ocupadas nessa faixa etária multiplicado por 100" (PNUD, 2010, n.p).

8. Tamanho do mercado - Proporção de pobres 2010, dado pela "proporção dos indivíduos com renda domiciliar per capita igual ou inferior a $\mathrm{R} \$ 140,00$ mensais, em reais de agosto de 2010" (PNUD, 2010, n.p). A variável foi tida como uma proxy do tamanho do mercado considerando a sua parcela com menor acesso.

As etapas operacionais necessárias ao cálculo do ICRM-A podem ser assim definidas: i) compilação da base de dados estatísticos; ii) cálculo dos quartis, mínimo e máximo para cada uma das variáveis explicativas; iii) identificação das variáveis explicativas quanto a sua relação com a competitividade regional, se direta ou inversa; iv) classificação dos dados municipais; v) pontuação conforme critério predeterminado; e, v) somatória de pontos.

\section{Resultados e discussão}

O Brasil tem uma população estimada em mais de 211 milhões de habitantes espalhados em um território de dimensões continentais. As regiões que concentram o maior número de habitantes proporcionais são: Sudeste (42\%); Nordeste (27,1\%); Sul (14,3\%); Norte $(8,8 \%)$; e, Centro-Oeste (7,8\%) (IBGE, 2020).

Em 2010, existiam 5.565 municípios no Brasil (nos dias atuais são 5.570) distribuídos nas cinco regiões já citadas e estas em 27 Unidades Federativas, que são: i) Centro-Oeste (Mato Grosso do Sul, Mato Grosso, Goiás e Distrito Federal); ii) Nordeste (Maranhão, Piauí, Ceará, Rio Grande do Norte, Paraíba, Pernambuco, Alagoas, Sergipe e Bahia); iii) Norte (Rondônia, Acre, Amazonas, Roraima, Pará, Amapá e Tocantins); iv) Sudeste (Minas Gerais, Espírito Santo, Rio de Janeiro e São Paulo); v) Sul (Paraná, Santa Catarina e Rio Grande do Sul) (IBGE, 2020).

O cálculo do ICRM-A definiu uma pontuação para cada um dos municípios brasileiros que obteve um mínimo de 1 e um máximo de 27. A figura 1 retrata o mapa dos municípios do Brasil e seu nível de ICRM-A em uma escala de cores divergentes em que o amarelo mais claro representa menores níveis cuja transição para maiores níveis se dá através do escurecimento passando por um tom arroxeado até um azul escuro. Os municípios em cinza claro são os criados posteriormente a 2010 . 
Figura 1 - Mapa com o ICRM-A dos 5.565 municípios brasileiros, ano 2010.

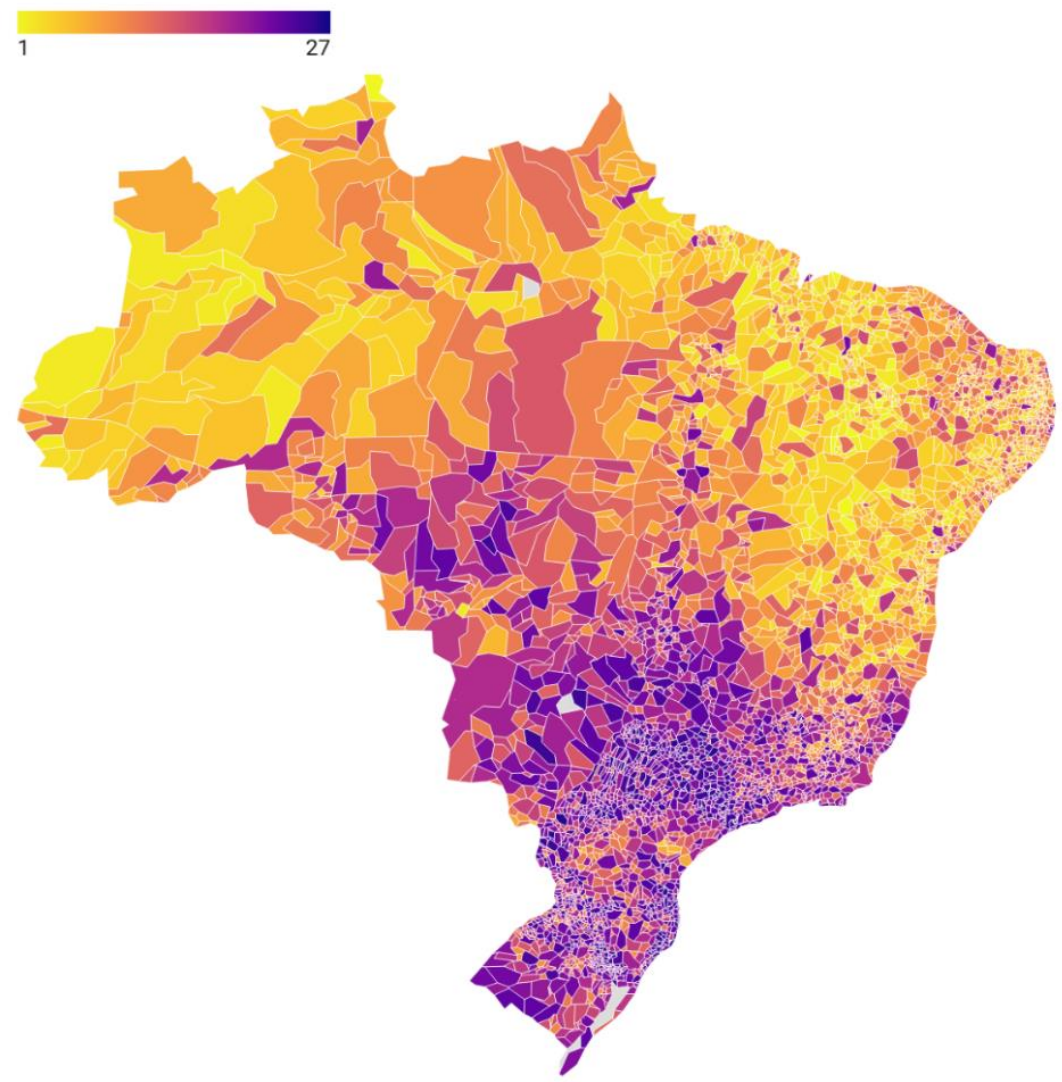

Fonte: Elaborado pelos autores.

É possível observar uma predominância de baixos níveis de competitividade regional municipal nas regiões norte e nordeste, bem como níveis mais altos de competitividade nas regiões centro-oeste, sudeste e sul. Municípios mais próximos ao litoral (leste), visualmente, possuem extensões territoriais menores que os localizados a oeste, provavelmente em uma particularidade criada durante o processo de colonização.

A figura 2 agrupa os municípios conforme classe de competitividade organizada em quatro, que são: Baixa (menor que 6,75); Média (entre 6,76 e 13,5); Alta (entre 13,6 e 20,25); e, Muito Alta (entre 20,26 e 27). O ICRM-A e o IDH-M mostraram correlação muito forte $(r=0,940)$, com intervalo de confiança de $95 \%(0,937$ e 0,943$)$ e valor- $p=0,000$, portanto, estatisticamente significante. Existem variáveis comuns entre as duas métricas, como RDPC (Renda Domiciliar Per Capita) e a ESPVIDA (Esperança de vida ao nascer). Essa evidência sugere que a competitividade regional em seus níveis iniciais se aproxima do conceito de desenvolvimento, e em níveis mais avançados (prontidão tecnológica, sofisticação de negócios e inovação) é que se perfaz a sua perspectiva de explicar o desenvolvimento potencial dado pelo cerne do conceito da competitividade regional. 
Figura 2 - Mapa com o ICRM-A dos 5.565 municípios brasileiros agrupados em quatro classes, ano 2010.

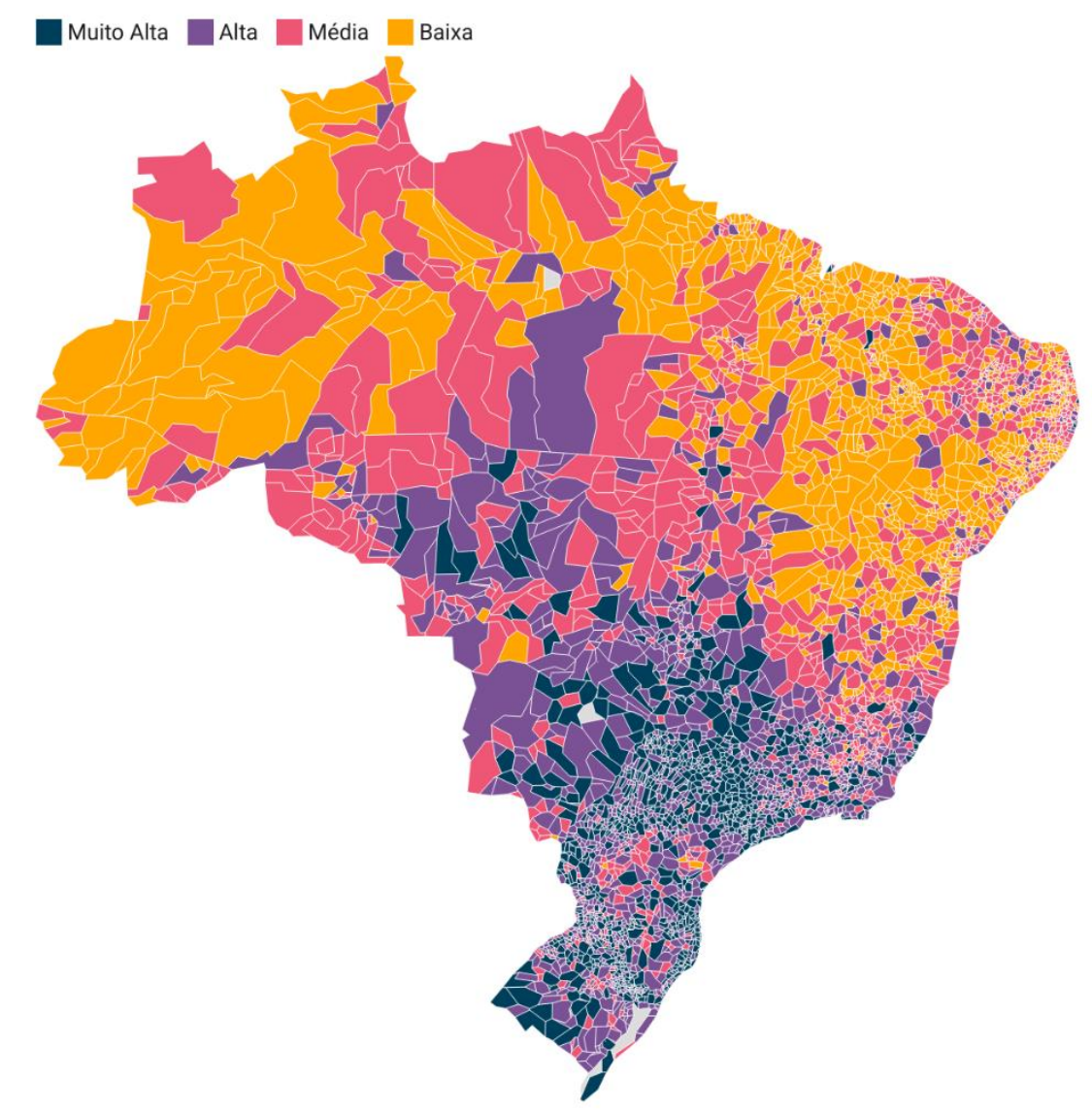

Fonte: Elaborado pelos autores.

O maior ICRM-A, com 27 pontos, foi da cidade de São Caetano do Sul (SP), com o 47은 maior PIB; 10 maior Renda Domiciliar Per Capita; 100\% dos domicílios com iluminação proveniente de luz elétrica; 210 maior esperança de vida ao nascer; 404으 Expectativa de anos de estudo aos 18 anos de idade; 4 ㅇ maior percentual da população de 25 anos ou mais com superior completo; 20 maior percentual de ocupados de 18 anos ou mais que são empregadores; e, uma das menores proporção de pobres do país (5.549ㅇ). Também é o município com o maior IDH-M 2010 dentre todos os municípios avaliados no período. Com 26 pontos no ICRM-A estão 39 municípios, podendo-se citar entre eles: João Monlevade, Altinópolis, Holambra, Lucélia, Santa Fé do Sul, Maringá, Balneário Camboriú, Jaraguá do Sul, Gramado e Nova Prata.

Entre os piores ICRM-A do país, com 51 municípios empatados com apenas 1 ponto, estão: Uiramutã, São João da Ponta, Montes Altos, Satubinha, Alagoinha do Piauí, Caxingó, Jurema, Pau D’Arco do Piauí, Pavussu, Queimada Nova, Curral de Cima, São José de Caiana, Salgadinho, Gentio do Ouro, Itaquara, Lamarão, Pedra Bonita e Santa Helena de Minas. O pior 
IDH-M do Brasil, da cidade de Melgaço (PA), está com 3 pontos do ICRM-A e na posição 5.122ㅇ․ A análise das frequências confirma a percepção visual do mapa.

No quadro 2 estão consolidadas as distribuições dos municípios quanto aos seus níveis de ICRM-A por região e Unidade Federativa. A distribuição dos níveis da competitividade regional dos municípios (Baixa, Média, Alta e Muito Alta) respectivamente, se distribuem da seguinte forma: i) Centro-Oeste (1,72\%; 30,04\%; 52,58\%; e, 15,67\%); ii) Nordeste (57,75\%; 36,57\%; 5,13\%; e, 0,56\%); iii) Norte (40,98\%, 48,78\%, 9,13\%; e, 1,11\%); iv) Sudeste (4,80\%; 23,38\%; 40,47\%; e, $31,35 \%)$; v) Sul (0,34\%; 13,47\%; 46,97\%; e, 39,23\%). É importante observar que o número de municípios das regiões também é bem diferente: Centro-Oeste (466; 8,37\%); Nordeste (1.794; $32,24 \%) ;$ Norte (449; 8,07\%); Sudeste (1.668; 29,97\%); e, Sul (1.188; 21,35\%).

Quadro 2 - Classificação do ICRM-A 2010 dos municípios por Unidade Federativa.

\begin{tabular}{|c|c|c|c|c|c|c|}
\hline \multirow[b]{2}{*}{ Região } & \multirow[b]{2}{*}{ UF } & \multirow[b]{2}{*}{ № Mun. } & \multicolumn{4}{|c|}{ ICRM-A } \\
\hline & & & $\begin{array}{c}\text { Baixo } \\
(1-6,75)\end{array}$ & $\begin{array}{c}\text { Médio } \\
(6,76-13,5)\end{array}$ & $\begin{array}{c}\text { Alto } \\
(13,6-20,25)\end{array}$ & $\begin{array}{l}\text { Muito alto } \\
(20,26-27)\end{array}$ \\
\hline \multirow{4}{*}{$\begin{array}{l}\text { CENTRO- } \\
\text { OESTE }\end{array}$} & MS & 78 & 1 & 17 & 37 & 23 \\
\hline & MT & 141 & 3 & 61 & 64 & 13 \\
\hline & GO & 246 & 4 & 62 & 144 & 36 \\
\hline & DF & 1 & - & - & - & 1 \\
\hline \multirow{9}{*}{ NORDESTE } & MA & 217 & 161 & 48 & 7 & 1 \\
\hline & $\mathrm{PI}$ & 224 & 185 & 34 & 4 & 1 \\
\hline & CE & 184 & 56 & 114 & 14 & - \\
\hline & RN & 167 & 63 & 91 & 11 & 2 \\
\hline & PB & 223 & 123 & 91 & 7 & 2 \\
\hline & $P E$ & 185 & 66 & 96 & 20 & 3 \\
\hline & $\mathrm{AL}$ & 102 & 70 & 29 & 3 & - \\
\hline & SE & 75 & 30 & 41 & 3 & 1 \\
\hline & BA & 417 & 282 & 112 & 23 & - \\
\hline \multirow{7}{*}{ NORTE } & RO & 52 & 4 & 32 & 15 & 1 \\
\hline & $A C$ & 22 & 11 & 10 & 1 & - \\
\hline & AM & 62 & 44 & 17 & 1 & - \\
\hline & $\mathrm{RR}$ & 15 & 5 & 9 & 1 & - \\
\hline & $\mathrm{PA}$ & 143 & 86 & 49 & 8 & - \\
\hline & AP & 16 & 5 & 9 & 2 & - \\
\hline & TO & 139 & 29 & 93 & 13 & 4 \\
\hline \multirow{4}{*}{ SUDESTE } & MG & 853 & 80 & 337 & 319 & 117 \\
\hline & ES & 78 & - & 14 & 50 & 14 \\
\hline & RJ & 92 & - & 12 & 57 & 23 \\
\hline & $\mathrm{SP}$ & 645 & - & 27 & 249 & 369 \\
\hline \multirow{3}{*}{ SUL } & PR & 399 & 3 & 57 & 206 & 133 \\
\hline & SC & 293 & - & 30 & 103 & 160 \\
\hline & RS & 496 & 1 & 73 & 249 & 173 \\
\hline
\end{tabular}

Fonte: Dados da pesquisa. 
A figura 3 possibilita averiguar as regiões com foco na média municipal em relação as 8 dimensões destacadas. Para fins de facilitar a análise visual, o percentual de pobres foi substituído pelo inverso do percentual de pobres, portanto, o gráfico do tipo radar pode ser interpretado que quanto mais "aberto" maiores são as médias municipais, consequentemente, maiores são seus indicativos de mais altos níveis de competitividade.

A região Sudeste se destaca tanto em relação as outras quanto a maiores médias do PIB e o inverso do percentual de pobres, ou seja, possui o menor percentual de pobres médio em relação as demais regiões). A região Sul tem destaque nas melhores médias quanto a esperança de vida ao nascer, expectativa de anos de estudo aos 18 anos de idade, percentual da população de 25 anos ou mais com superior completo, renda per capita média, percentual de ocupados de 18 anos ou mais que são empregadores e percentual da população que vive em domicílios com iluminação proveniente de energia elétrica.

Figura 3 - Gráfico tipo radar com a média padronizada das 8 variáveis explicativas do ICRM-A selecionadas, ano 2010.

$\longrightarrow$ CENTRO-OESTE NORDESTE NORTE SUDESTE $\longrightarrow$ SUL

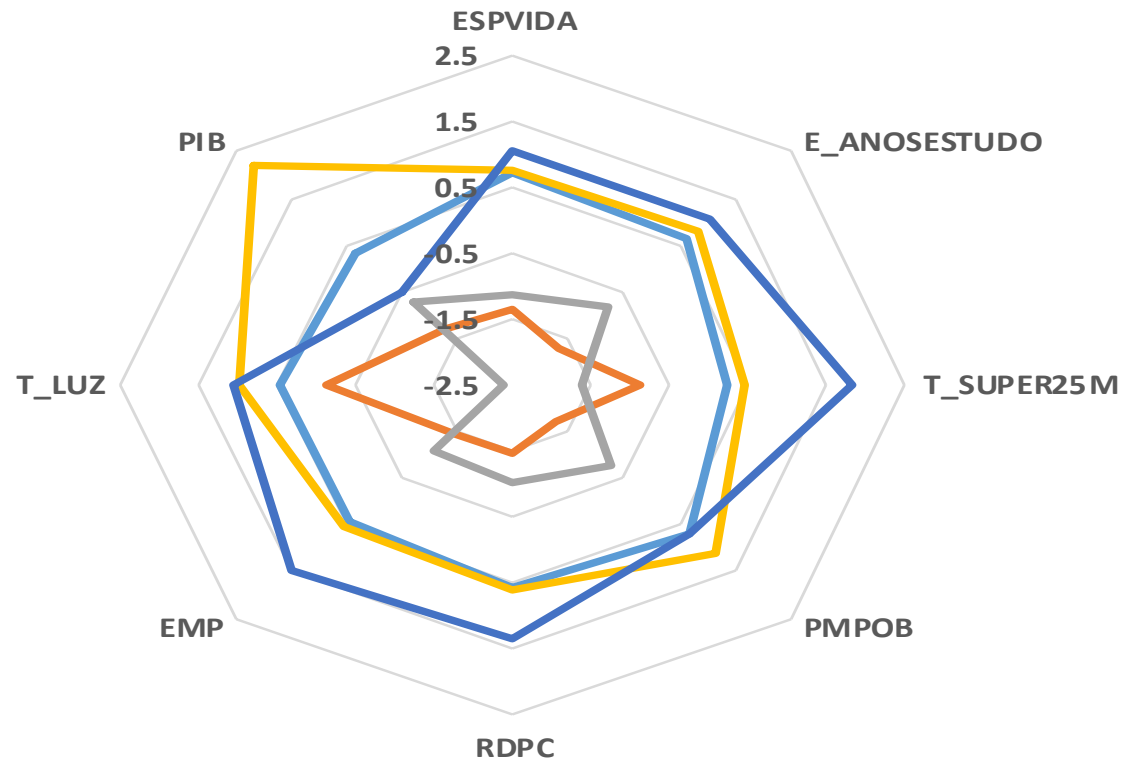

Fonte: Elaborado pelos autores.

A região Centro-Oeste ficou em uma posição intermediária em relação as demais e as piores médias dentre os indicadores foram divididas entre a região Norte e Nordeste. A região Norte apresentou as piores médias em relação a T_LUZ e T_SUPER25M, enquanto a região Nordeste os valores mais baixos em ESPVIDA, E-ANOSESTUDO, PMPOB, RDPC e EMP. É 
importante destacar que a região Centro-Oeste provavelmente foi influenciada positivamente pelo Distrito Federal, que possui uma realidade bem diferente do restante da região.

A figura 4 é um gráfico tipo boxplot (diagrama de caixa) que sintetiza algumas informações relevantes da estatística descritiva. O ICRM-A médio das regiões foi a variável selecionada. A média das regiões é representada pela linha vertical azul escura. A linha horizontal azul e fina representa o desvio padrão. A área destacada em azul representa os valores entre o primeiro e o terceiro quartil e a mediana (ou segundo quartil) é dado pela linha vertical amarela.

É possível verificar que a região Nordeste, acompanhada de perto da região Norte, possui as piores condições competitivas. As regiões Centro-Oeste, Sudeste e Sul encontram-se em um patamar superior. As médias, entretanto, escondem bolsões e pontos em que as regiões que vão na contramão do seu entorno, tanto com áreas de baixa competitividade no sul como de altas competitividades no norte.

Figura 4 - Gráfico tipo Boxplot para o ICRM-A das regiões, 2010.

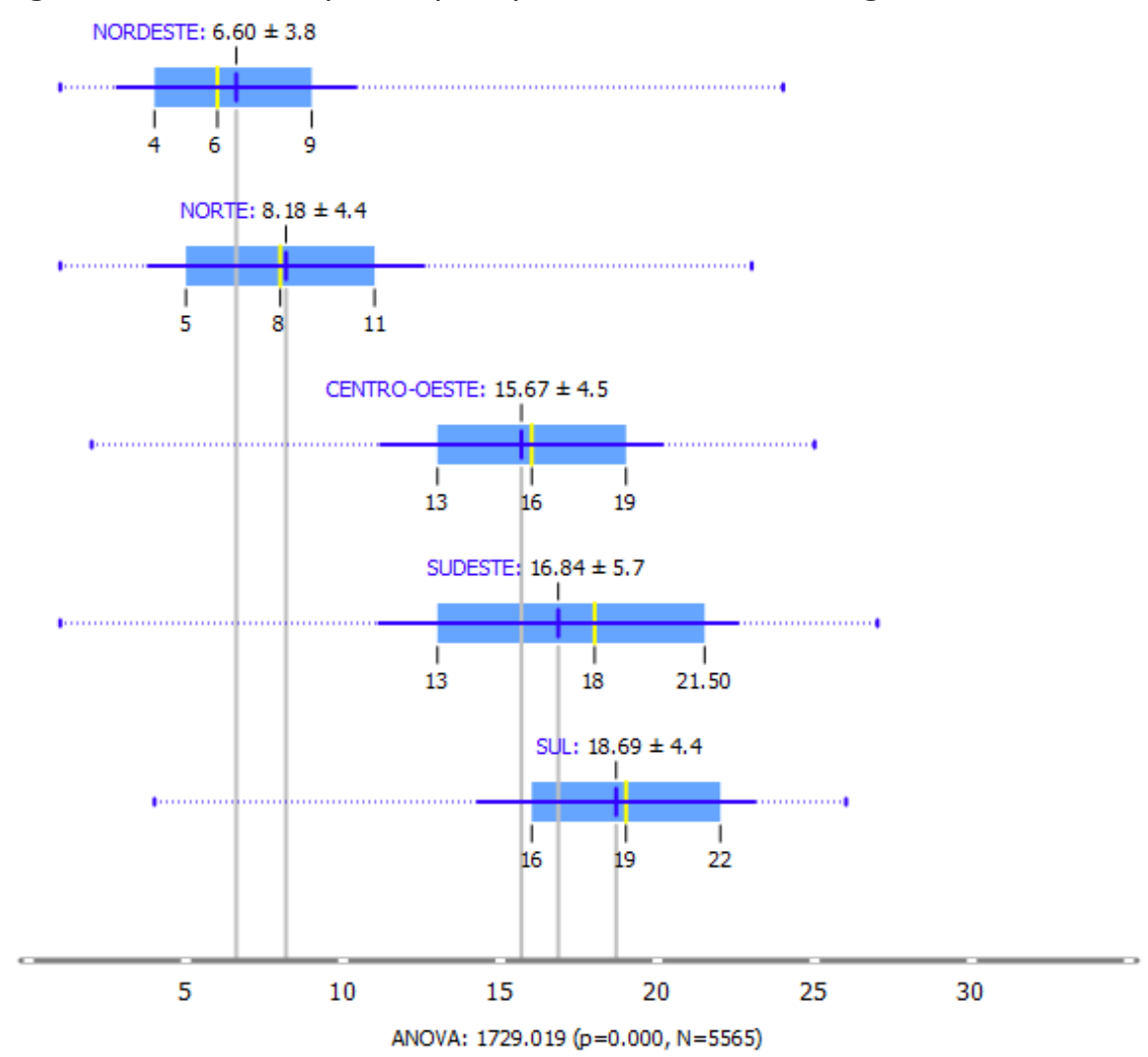

Fonte: Elaborado pelos autores.

Na figura 5 um algoritmo de árvore de regras (tree) foi utilizado para buscar padrões e regras dentre as variáveis. A maior classe é a alta, com 1.611 municípios (28,9\%) e a variável que melhor divide o conjunto das classes é a RDPC (Renda Domiciliar per capita), cujos valores 
menores ou igual a $R \$ 466,69$ separam muito bem a baixa e média competitividade da alta e muito alta. A Alta Competitividade tem ainda RDPC mais elevados ( $\mathrm{R} \$ 650,57$ ) que a alta, bem como percentual da população de 25 anos ou mais com superior completo (>6,76\%). A baixa e média competitividade também se separam ainda mais pela RDPC (baixa $\leq \mathrm{R} \$ 283,59$ e média > $\mathrm{R} \$ 283,59)$.

Figura 5 - Árvore de regras das 8 variáveis selecionadas tendo por variável meta as classes do ICRM-A.

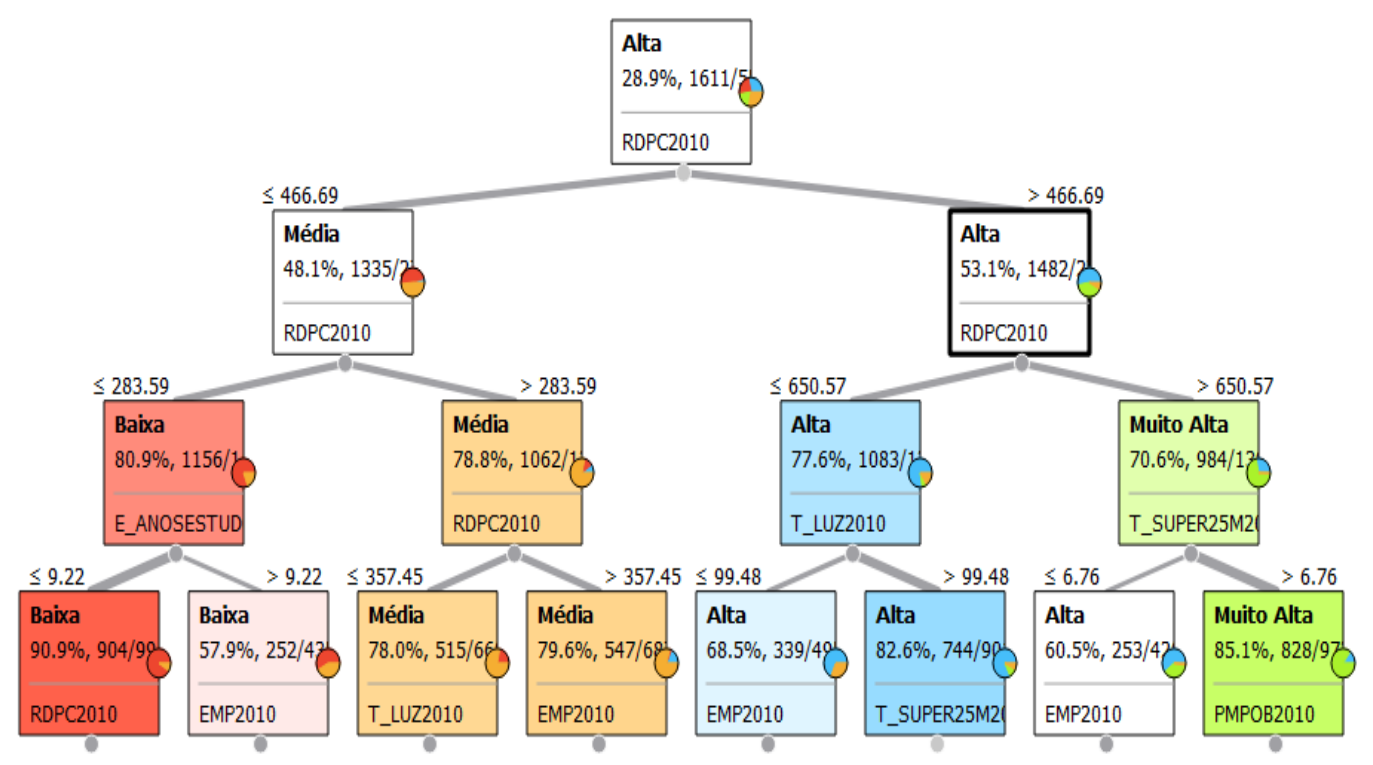

Fonte: Elaborado pelos autores.

Os resultados do ICRM-A trazem algumas constatações, como a heterogeneidade regional em relação ao nível de competitividade regional, com grande assimetria de condições entre as regiões mais ao sul e as mais ao norte. Também se sugere que os níveis preliminares da competitividade regional são altamente relacionados com o desenvolvimento e que a economia, no método representada pela RDPC, é um dos principais determinantes do ICRM-A. A competitividade regional respaldada na inovação e nos níveis mais elevados da competitividade, apesar dos enormes desafios relacionados ao estudo em municípios brasileiros, deve trazer insights sobre uma competitividade mais relacionada com a visão do potencial de desenvolvimento.

\section{Considerações finais}


O problema ao qual esse artigo buscou abordar foi como mensurar e classificar a competitividade regional em municípios brasileiros? A adaptação do Índice de Competitividade Regional Municipal (ICRM) de Dresch (2021), resultando no ICRM-A, uma versão que reduziu de 3 grupos, 11 subgrupos e 52 variáveis para 2 grupos, 8 subgrupos e 8 variáveis. 0 aumento da abrangência, de 79 municípios para 5.565, foi adequadamente resolvido com a adaptação, entretanto foi possível constatar uma perda de informação em relação aos níveis mais elevados da competitividade regional.

O ICRM-A evidenciou a heterogeneidade inter-regional, com condições menos favoráveis as regiões Norte e Nordeste em relação a Centro-Oeste, Sudeste e Sul. Pontos e bolsões de competitividade, ou da sua ausência, aparecem em todas as regiões e deveriam ser estudados com aprofundamento para proposições de políticas focalizadas voltadas ao desenvolvimento regional.

A variável RDPC, Renda Domiciliar per capita, mostrou ser bastante relevante na divisão entre os municípios mais e menos competitivos, portanto, mesmo que superada visão unicamente econômica, ela não pode ser ignorada de forma alguma. O trabalho é uma proposta exploratória, inicial e ainda incipiente, que pode e deve ser expandida e aperfeiçoada. As mais relevantes limitações do trabalho se relacionam a falta de descritores dos níveis mais elevados da competitividade regional (inovação, sofisticação de negócios e prontidão tecnológica) e o aprofundamento relacionado as manchas e pontos em que o ICRM-A não segue a tendência das suas regiões.

Como sugestão para trabalhos futuros fica a investigação pormenorizada da difusão de plataformas mais elevadas da competitividade regional e como estas se relacionam com políticas públicas e outras características endógenas das regiões. Também a discussão aprofundada entre as diferenças entre o desenvolvimento e a competitividade regional, bem como um estudo multicasos envolvendo municípios e clusters que possuem realidades muito diferentes do seu entorno.

\section{Referências}

AIGINGER, K. Competitiveness: from a dangerous obsession to a welfare creating ability with positive externalities. Journal of industry, competition and trade, v. 6, n. 2, p. 161-177, 2006. ANNONI, P.; DIJKSTRA, L. Measuring and monitoring regional competitiveness in the European Union. In: HUGGINS, R.; THOMPSON, P. Handbook of regions and competitiveness, 
contemporary theories and perspectives on economic development. UK: Edward Elgar Publishing Limited, 2017. p. 49-79.

BOROZAN, $Ð$. Regional competitiveness: Some conceptual issues and policy implications. Interdisciplinary Management Research, v. 4, n. 1, p. 50-63, 2008.

BRASIL. planalto.gov.br/. Constituição da República Federativa do Brasil de 1988, 2021. Disponivel em:

<http://www.planalto.gov.br/ccivil_03/constituicao/ConstituicaoCompilado.htm>. Acesso em: 4 Abril 2021.

DRESCH, L. D. O. Competitividade Regional em municípios brasileiros: uma proposta metodológica de classificação e análise baseada na Design Science, 248 f. (Doutorado em Administração) - Curso de Pós-Graduação em Administração, Universidade Federal de Mato Grosso do Sul, Campo Grande, 2021.

HUGGINS, R.; THOMPSON, P. Introducing regional competitiveness and development: contemporary theories and perspectives. In: HUGGINS, R.; THOMPSON, P. Handbook of Regions and Competiveness: Contemporary Theories and Perspectives on Economic Development. Cheltenham: Edward Elgar, 2017. p. 1-31.

IBGE. www.ibge.gov.br/. IBGE Cidades e Estados, 2020. Disponivel em: <https://www.ibge.gov.br/cidades-e-estados.html>. Acesso em: 20 Abril 2021.

PNUD. Atlas do Desenvolvimento Humano no Brasil, 2010. Disponivel em: <http://atlasbrasil.org.br/2013/pt/>. Acesso em: 1 Abril 2021.

PORTER, M. The economic performance of regions. Regional studies, v. 37, n. 6-7, p. 549-578, 2003.

PORTER, M. On competition. [S.I.]: Harvard Business Press, 2008.

SPIEGEL, M. R. Estatística. São Paulo: Makron Books, 1993.

STORPER, M. The regional world: territorial development in a global economy. [S.I.]: Guilford press, 1997. 